$(p=0.03)$ but not on Form Constancy $(p=0.17)$. Compared to VLBW children without any oculomotor or visual sensory deficit, VLBW children with one or more of these deficits performed worse only one visual perceptive measure (Figure-Ground perception; $p=.01$ ).

Discussion We found reduced functioning in VLBW children for binocularity, perceptual grouping, visual-spatial judgment and figure-ground segmentation. Except for figure-ground segmentation, these visual perceptive deficits remain present in the absence of oculomotor and sensory deficits.

\section{ANTENATAL FACTORS ASSOCIATED WITH DEVELOPMENTAL DELAY IN MODERATELY PRETERMI-BORN CHILDREN, RESULTS OF A COHORT STUDY}

doi:10.1136/archdischild-2012-302724.0327

IJ Kerstjens, ${ }^{2} \mathrm{AF}$ Winter de, ${ }^{3} \mathrm{KM}$ Sollie, ${ }^{2} \mathrm{MR}$ Potijk, 'IF BoccaTjeertes, ${ }^{2}$ SA Reijneveld, ${ }^{1} \mathrm{AF}$ Bos, Lollipop. 'Neonatology, Beatrix Children's Hospital, University of Groningen, University Medical Center Groningen; ${ }^{2}$ Health Sciences, University Medical Center Groningen, University of Groningen; ${ }^{3}$ Obstetrics, University of Groningen, University Medical Center Groningen, Groningen, The Netherlands

Background Worldwide 6-9\% of all children are born moderately preterm $\left(32^{+0}-35^{+6}\right.$ weeks' gestation). They are at risk for developmental delay in early childhood. Knowledge on the influence of antenatal maternal, fetal, and delivery-related factors on the development of moderately preterm-born children is limited.

Objective To determine the association between developmental delay in early childhood and antenatal factors in moderately preterm-born children.

Design/methods We measured development with the Ages and Stages Questionnaire (ASQ) at age 43-49 months in 834 moderate preterms born in 2002-2003, in a community-based cohort study.

A total ASO score $>2$ SD below the Dutch mean reference was considered to indicate developmental delay. Data on maternal, fetal, and delivery-related factors were obtained from medical records. We used logistic regression to estimate odds ratios (ORs) for developmental delay, adjusted for socio-demographic variables.

Results In univariate analyses, several fetal and maternal factors were associated with risk of developmental delay. In multivariate analyses, only pre-existent obesity (odds ratio (OR) 3.0, 95\% confidence interval (CI): 1.5-5.8), multiparity (OR:2.8, CI: 1.6-4.9), Small-for-gestational-age (SGA) (OR:2.9, CI: 1.4-6.1), multiple pregnancy (OR:1.8, CI: 1.0-3.3), and male gender (OR:4.1, CI: 2.2-8.6) increased risk of developmental delay.

Conclusions Of all antenatal factors studied, no modifiable factors were associated with developmental delay except for SGA. Enhanced prevention of intra-uterine growth restriction, interventions aiming at reducing pre-pregnancy weight in fertile women, and reducing number of transferred embryos in assisted reproduction might offer routes to improve developmental outcomes in children eventually born moderately preterm.

\section{VALIDATION OF ERIC - A NEW PARENTAL REPORT INSTRUMENT FOR DETECTION OF COGNITIVE DELAY IN AT-RISK INFANTS}

doi:10.1136/archdischild-2012-302724.0328

${ }^{1} \mathrm{G}$ Schafer, ${ }^{1} \mathrm{~L}$ Genesoni, ${ }^{2} \mathrm{R}$ Jones, ${ }^{3} \mathrm{HA}$ Doll, ${ }^{4} \mathrm{E}$ Adams, ${ }^{5} \mathrm{R}$ Gray, ${ }^{6} \mathrm{G}$ Boden. ${ }^{1}$ Department of Psychology, University of Reading, Reading; ${ }^{2}$ Paediatric Department, Wexham Park Hospitals, Slough; ${ }^{3}$ Department of Population Health, Norwich Medical School, Norwich; ${ }^{4}$ Neonatal Unit, John Radcliffe Hospital; ${ }^{5}$ National Perinatal Epidemiology Unit (NPEU), University of Oxford, Oxford; ${ }^{6}$ Neonatology, Royal Berkshire Hospital, Reading, UK

Background and Aims At 2 years, cognitive delay is the most common form of developmental disability in the preterm population (Marlow, 2004). We have established the diagnostic properties of a new cognitive developmental screen (Early Report by Infant Caregivers, ERIC), between 10-24 months.
Methods Participants. 362 infants aged 10-24 months, with at least one of: weight $<1500 \mathrm{~g},<34$ completed weeks gestational age, 5 -min Apgar $<7$, HIE. Children with impairments preventing fair assessment by ERIC were excluded. Parents/caregivers completed ERIC at home before administration of the Cognitive Scale of the Bayley Scales of Infant Development III. Delay was defined as a prematurity-corrected Bayley score $<80$ (Moore et al, 2011).

Results Nineteen infants were delayed, with age-corrected ERIC scores lower than those without delay $(\mathrm{p}<0.001)$. On ROC analysis, Area Under the Curve was 0.86 , with $83 \%$ sensitivity (95\% CI 66-99.9\%), 79\% specificity (75-83\%), 19\% Positive Predictive Value (PPV) (2-36\%), and 98\% Negative Predictive Value (NPV) (96-99.6\%). The low PPV reflects low prevalence of delay (5.2\%) in this sample.

Conclusions ERIC provides a useful diagnostic screening tool, able to rule out developmental delay in this population (NPV $=98 \%$ ).

Note This abstract presents independent research funded by the National Institute for Health Research (NIHR) under its Research for Patient Benefit (RfPB) Programme (Grant Reference PB-PG0807-14202). The views expressed are those of the authors and not necessarily those of the NHS, the NIHR or the Department of Health. Preliminary results were presented to the Neonatal Society, London, March 2012.

\section{SEVERE RETINOPATHY OF PREMATURITY (ROP) REMAINS A MARKER OF CHILDHOOD DISABILITIES: RESULTS FROM THE CAFFEINE FOR APNEA OF PREMATURITY TRIAL}

doi:10.1136/archdischild-2012-302724.0329

${ }^{1,2} \mathrm{~B}$ Schmidt, ${ }^{3} \mathrm{P}$ Davis, ${ }^{3} \mathrm{~L}$ Doyle, ${ }^{3} \mathrm{P}$ Anderson, ${ }^{4} \mathrm{E}$ Asztalos, ${ }^{5} \mathrm{~A}$ Solimano, ${ }^{5} \mathrm{R}$ Grunau, ${ }^{4} \mathrm{~A}$ Ohlsson, ${ }^{6} \mathrm{D}$ Dewey, ${ }^{7} \mathrm{D}$ Moddemann, ${ }^{8} \mathrm{~K}$ Barrington, ${ }^{9} \mathrm{~W}$ Tin, ${ }^{2} \mathrm{R}$ Roberts, for the Caffeine for Apnea of Prematurity (CAP) Trial Investigators. 'University of Pennsylvania, Philadelphia, PA, USA; ${ }^{2}$ McMaster University, Hamilton, ON, Canada; ${ }^{3}$ University of Melbourne, Melbourne, VIC, Australia; ${ }^{4}$ University of Toronto, Toronto, ON; ${ }^{5}$ University of British Columbia, Vancouver, BC; ${ }^{6}$ University of Calgary, Calgary, $A B$; ' University of Manitoba, Winnipeg, MB; ${ }^{8}$ University of Montreal, Montréal, OC, Canada; ${ }^{9} \mathrm{James}$ Cook University, Middlesbrough, UK

Background The Cryotherapy for Retinopathy of Prematurity Cooperative Group showed that the severity of ROP was a marker for functional disability at 5.5 years in infants $\leq 1250 \mathrm{~g}$ BW who were born in the late 1980s.

Objective To determine whether severe ROP remains a strong predictor of visual and non-visual disabilities at age 5 years in infants $\leq 1250$ g BW who were enrolled in the CAP trial between 1999 and 2004.

Methods 5-year follow up of 1580 surviving CAP children with known ROP status. Severe ROP was defined as stage 4 or 5 disease or receipt of retinal therapy in at least one eye. Outcomes were disabilities in 6 domains, and including cognitive impairment (Full Scale IQ< 70), motor impairment (GMFCS level 2-5), deafness and blindness. Odds ratios were adjusted for antenatal steroids, gestational age, sex, multiple birth, and mother's education.

Results There were 94 survivors with and 1486 without severe ROP. Rates of visual and non-visual disabilitites were significantly higher in children with severe ROP (Table 1).

Abstract 329 Table 1

\begin{tabular}{lcccc}
\hline Outcome & Severe ROP & No Severe ROP & Adjusted OR & 95\% CI \\
\hline IO $<70$ & $15 \%$ & $4.5 \%$ & 4.0 & 1.9 to 8.4 \\
Motor impairment & $13 \%$ & $2.4 \%$ & 4.0 & 1.9 to 8.6 \\
Deafness & $13 \%$ & $2.4 \%$ & 4.0 & 1.9 to 8.6 \\
Blindness & $14 \%$ & $0.1 \%$ & 129 & 21 to 786 \\
Any disability & $45 \%$ & $16 \%$ & 3.5 & 2.2 to 5.6 \\
\hline
\end{tabular}


Conclusions Severe ROP remains a strong marker of childhood disabilities in a recent and large international cohort of infants $\leq 1250$ g BW.

\section{THE ASSOCIATION BETWEEN PRETERM BIRTH AND AUTISM COULD BE EXPLAINED BY MATERNAL AND NEONATAL MORBIDITY}

doi:10.1136/archdischild-2012-302724.0330

S Johansson, S Buchmayer, A Johansson, C Hultman, P Sparen, S Cnattingius. Karolinska Institutet, Stockholm, Sweden

Background and Aims Children born preterm face an increased risk of autistic disorders.

We examined whether the association between preterm birth and risk of autistic disorders could be explained by pregnancy complications or neonatal morbidity.

Methods Swedish, population-based, case-control study including 1216 cases with autistic disorders born between 1987 and 2002, and 6080 controls matched with respect to gender, birth year, and birth hospital. Associations between gestational age and autistic disorders were assessed and adjusted for maternal, birth and neonatal characteristics.

Results Compared with infants born at term, the unadjusted odds ratios (ORs) for autistic disorders among very and moderately preterm infants were 2.05 [95\% CI: $1.26-3.34$ ] and 1.55 [95\% CI: 1.22 $1.96]$, respectively.

In analyses controlled for maternal, pregnancy, and birth characteristics, ORs were reduced to 1.48 [95\% CI: 0.77-2.84] and 1.33 [95\% CI: 0.98-1.81], respectively.

Adding also neonatal complications to the analyses, ORs were 0.98 [95\% CI: 0.45-2.16] and 1.25 [95\% CI: 0.90-1.75], respectively.

Reductions in risks of autistic disorders related to preterm birth were primarily attributable to preeclampsia, small-for-gestational age birth, congenital malformations, low Apgar scores at 5 minutes, and intracranial bleeding, cerebral edema, or seizures in the neonatal period. Neonatal hypoglycemia, respiratory distress, and neonatal jaundice were associated with increased risk of autistic disorders for term but not preterm infants.

Conclusions The increased risk of autistic disorders related to preterm birth is mediated primarily by prenatal and neonatal complications that occur more commonly among preterm infants.

\section{THE PROGNOSTIC VALUE OF AEEG AND NIRS DURING THERAPEUTIC HYPOTHERMIA IN TERM ASPHYXIATED NEWBORNS}

doi:10.1136/archdischild-2012-302724.0331

${ }^{1} \mathrm{C}$ Niezen, ${ }^{1} \mathrm{~A}$ Bos, ${ }^{2} \mathrm{D}$ Sival, ${ }^{1} \mathrm{H}$ ter Horst. ${ }^{1}$ Pediatrics; ${ }^{2}$ Child Neurology, University Medical Center Groningen, Groningen, The Netherlands

Background and objective Infants with hypoxic-ischemic encephalopathy (HIE) are treated with therapeutic hypothermia (HT). Following perinatal asphyxia amplitude-integrated EEG (aEEG) and near-infrared spectroscopy (NIRS) are used to determine prognosis. We aimed to assess the prognostic value of aEEG and NIRS during HT.

Methods 40 term infants with HIE and treatment with HT were retrospectively studied. aEEG and NIRS were started immediately following admission. aEEGs were assessed by pattern recognition: background pattern (BP), presence of sleep wake cycling (SWC) and epileptic activity (EA) were appraised. Recordings during HT (72 hrs) were analysed.

Results $84 \%$ of infants had an abnormal BP (discontinuous normal voltage, burst suppression (BS), continuous low voltage (CLV) or flat trace $(\mathrm{FT}))$ at admission. The $\mathrm{LR}+(95 \% \mathrm{CI})$ of an severely abnormal BP (BS, CLV, FT) for mortality was 1.97 (1.24-3.12) at $6 \mathrm{~h}$ after birth and increased to 4.5 (3.16-6.39) at 24h, $6.3(2.04-19.4)$ at $48 \mathrm{~h}$ and 6.19 (1.93-19.8) at 72h. LR+ of BS for mortality was below 1 at any time. LR+ of EA for mortality was 4.95 (2.20-11.1), the type of EA (e.g. status epilepticus) was not predictive. LR+ of SWC for survival was 10.7 (1.62-70). RcSO2 increased from 6 to $72 \mathrm{~h}$ after birth, but was not different at any time between infants that died or survived.

Conclusion aEEG during HT can still be used to predict risk for mortality of HIE, especially beyond $24 \mathrm{hrs}$. BS is frequently not associated with a fatal outcome. RcSO2 has no additional value to predict mortality.

\section{THE RELATIONSHIP BETWEEN RAPID FLUCTUATION IN SERUM SODIUM AND INTRAVENTRICULAR HEMORRHAGE (IVH) IN HYPERNATREMIC EXTREMELY LOW BIRTH WEIGHT PRETERM INFANTS}

doi:10.1136/archdischild-2012-302724.0332

S Sarkar, J Dalton, R Dechert. Department of Pediatrics, Division of Neonatal-Perinatal Medicine, University of Michigan Health System, Ann Arbor, MI, USA

Hypernatremia causes brain shrinkage and resultant vascular rupture with cerebral and IVH. However, it is not known if rapid fluctuation in serum sodium in hypernatremic preterm infants results in IVH or death

Objective To determine if the rapid rise in serum sodium or rapid correction of hypernatremia predict the composite outcome of severe IVH (grade 3 and 4) or death during the first 10 days of life.

Methods Single center retrospective review of 167 preterm infants with GA $\leq 26$ weeks who had serum sodium monitored at least every 12-24 hours and more frequently, if indicated. Logistic regression analysis identified which of the commonly cited risk factors of $\mathrm{IVH}$, including rapid ( $>10$ and $>15 \mathrm{mmol} / \mathrm{l} /$ day) rise or fall in serum sodium could predict composite outcome in hypernatremic infants. Results 98 (59\%) of 167 infants studied developed hypernatremia (serum sodium $>150 \mathrm{mmol} / \mathrm{L}$ ), with a maximum median serum sodium of $154 \mathrm{mmol} / \mathrm{l}$ (range 150-181, IOR 152-157), occurring on median postnatal age of 4 days (IOR 3-5). Grade 4 IVH was more frequent in hypernatremic compared to normonatremic infants $(p=0.032$, OR 3.4, 95\% CI 1.1-10.6). Among 98 infants with hypernatremia, severe IVH or death occurred in 33 and 21 infants with rapid ( $>10 \mathrm{mmol} / \mathrm{l} /$ day) rise and drop in serum sodium, respectively. However, rapid ( $>10$ and $>15 \mathrm{mmol} / \mathrm{l} /$ day) rise or fall in serum sodium was not associated with composite outcome on multivariate analysis.

Conclusion Correction of hypernatremia not exceeding 10 to 15 $\mathrm{mmol} / \mathrm{l} /$ day in hypernatremic preterm infants was not associated with severe IVH or death.

\section{AUTOMATIC IDENTIFICATION OF ACTIVITY BURSTS IN EEG OF PRETERM INFANTS}

doi:10.1136/archdischild-2012-302724.0333

K Murphy, R Goulding, N Stevenson, GB Boylan. Paediatrics and Child Health, University College Cork, Cork, Ireland

Background EEG monitoring provides important information about the neurological status of the preterm infant but is difficult to interpret for most. We aim to automatically detect the typical bursting pattern (trace discontinu) of the preterm EEG and compare the detections with expert manual annotations.

Methods The method was based on the single channel EEG method of Palmu et al. but extended to 8-channel recordings for the first time. The EEG signal was first filtered with a Kaiser-window filter and the output of a non-linear energy operator (NLEO) was calculated. The NLEO signal was smoothed and corrected for baseline artefacts. A burst was identified if the resulting signal remained 